\title{
3D Modeling By the Masses: A Mobile App for Modeling Buildings
}

\author{
Todd Eaglin and Kalpathi Subramanian and Jamie Payton \\ Department of Computer Science \\ The University of North Carolina at Charlotte, Charlotte, NC, USA \\ \{teaglin,krs,payton\}@uncc.edu
}

\begin{abstract}
Knowledge of the 3D geometry of large urban buildings and surrounding structures is important in indoor navigation applications, emergencies, and evacuation response. Visual representations of the 3D geometry can be useful to users navigating unfamiliar buildings. The traditional methods of constructing 3D models from CAD drawings or GIS systems are prone to error, difficult to modify in a timely manner, can easily become out-of-date, and are not scalable. We exploit the power of current mobile devices (smartphones, tablets) and their 3D graphics rendering capabilities to present a mobile application that uses crowdsourcing to build 3D models of large buildings and urban structures. Our system is based on a client-server architecture, where users of a mobile application create, submit, and vote on $3 \mathrm{D}$ models of building components; the server collects and uses votes pertaining to accuracy and completion of a model to determine if an object may be approved. We describe preliminary results from using the system on a floor of an academic building and propose its deployment and demonstration during Percom 2013 for mapping the conference venue by meeting participants.
\end{abstract} tion;

Keywords-3D geometric modeling; crowdsourcing; naviga-

\section{INTRODUCTION}

Models of 3D buildings are typically acquired from CAD drawings or designed using GIS systems and stored in databases. Most often, these tend to be in 2D with separate datasets for each floor of a building and additional attribute data held in different layers (structures). On the other hand, 3D interactive modeling has a long history, based on 3D interactive graphics systems and has been in common use in automobile, aerospace and numerous other engineering applications.

Access to 3D building data has numerous applications, including indoor navigation. Visual information about a building can be very useful in the hands of users navigating an unfamiliar building, especially for first responders in an emergency or evacuation (e.g., due to the presence of a fire, chemical exposure, or a threatening and armed individual). The proliferation of mobile computing devices (e.g., smartphones, tablets) and the availability of powerful 3D graphics rendering capabilities that is currently possible on this platform opens up new opportunities and applications that were not possible even a few years ago.

We present a 3D geometric modeler for interactive construction of building geometry on mobile devices. In addition to exploiting the graphics geometry processing and rendering power of today's mobile computers, we use crowdsourcing as the means to rapidly generate 3D models and voting, ranking, and approval procedure. The goal is to lead towards a building a 3D model of the internal structure of a building that is sufficiently accurate to serve navigation type applications, and that can be adapted for use in other applications. Our design and goals are somewhat similar to Wikipedia[8], in that our system attains data quality and accuracy by empowering users to repeatedly contribute, edit and check the validity of the contributed data.

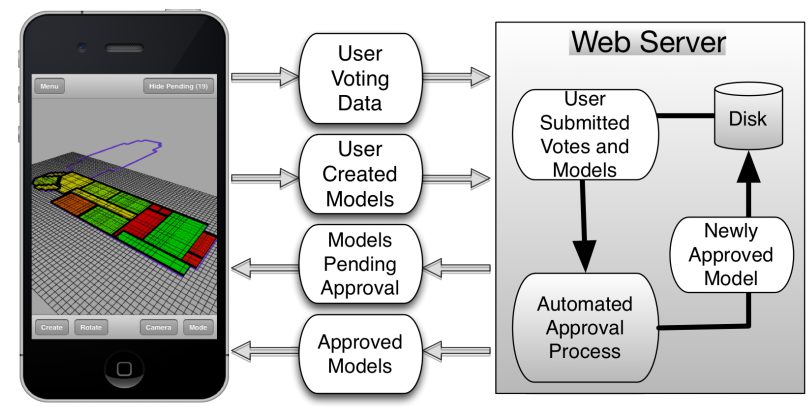

Figure 1. Mobile 3D Modeler System Architecture.

Related work in this regard includes the OpenStreetMap system [4]. Crowdsourcing in OpenStreetMap was a successful means of utilizing public efforts to effectively map data that would otherwise be extremely expensive. This work was expanded to mapping indoor locations in OpenRoomMap [7], exploring the potential and accuracy of mapping buildings. However, the authors encountered several issues and the system restricted the floor plans to maintain accuracy. OpenRoomMap utilized manually modified architectural drawings that outlined every aspect of the building. Users interacting with the system were unable to make modifications or alter these outlines in any way. Additions to the building structure, like the modifications of rooms, would be impossible through the current implementation. Lastly, the system also encountered vandalism through outside user participation.

Our own earlier work on constructing 3D visualizations of building structures for indoor navigation used CAD drawings as input to build a 3D graph structure of a building [5], [3]. We have used the system to model over 70 campus 
academic and student residence buildings. As mentioned above, modifications and updates to the model in this kind of approach can be tedious and most building drawings are out of date. Kim et al. [6] focused on use of mobile devices for situationally-aware emergency response and training. Woodman and Harley [9] focused on tracking padestrians within a building; they exploited sensors within mobile devices (compass, accelerometers) to accomplish this, however they assumed the existence of accurate building geometry. In contrast, our work does not rely on this assumption and instead harnesses the intelligence of the crowd to vote on the accuracy and completeness of user-generated models. Goodchild [2] surveys mapping techniques and concludes that crowdsourcing is a means to future mapping of urban spaces, given the decline and expense of commercial mapping; our work provides an implementation of this approach.

\section{The Mobile 3D ModeleR}

Fig. 1 illustrates the architecture of the mobile 3D modeler. The core of our design revolves around clientserver architecture. Mobile users (clients) use an interactive 3D modeling app on their mobile devices (currently on Apple iPhone/iPod/iPad devices) to construct components of a building. These can be rooms, hallways, furniture (chairs/tables, etc). The model can then be uploaded to a server for other users to access, augment and evaluate. Once these models are submitted by the user to the server, they are disseminated to users of the application to solicit votes on the created models. Votes may based on several criteria; currently we ask users to vote on completeness and accuracy of a submitted model. A model is considered to be complete if a room contains all existing components. The second measure is accuracy, which considers the dimensions and location of modeled objects (e.g., rooms, hallways, placement of furniture) as compared to the user's knowledge of the ground truth of the physical space. The server tabulates the votes from users and uses thresholds to determine whether or not to approve the model as a quality representation of the real world. The server uses a color scale (green through red) to communicate the status of the object to users who may see the object as either pending (unapproved) objects or approved objects (of sufficient quality). An example model of the first floor of an academic building as captured by a user of our mobile application is shown in Fig. 2. Here rooms in red are waiting for additional votes, while those in green have received sufficient votes for approval.

The mobile application allows users to create geometry through a simplified toolset and editor. The toolset includes navigation, object creation and object modification. The editor includes predefined objects for creating indoor building geometry. The editor uses a two dimensional grid scaled appropriately to match the dimensions of a building being mapped. This environment simplifies the creation of geometry for users as well it also provides unification to every model developed. This way users are always using the same scale and working in the same environment. Highly structured models (classrooms with uniformly distributed furniture, for instance) are easily created by interactively selecting multiple tiles (representing furniture locations) and simply instancing the object at all selected locations. Grouping, ungrouping and Copy/Paste operations further provide scalable means to create models efficiently with minimal interaction.

The server handles all communication between clients, but its primary task is running an automated approval process. It works in several steps. First, users generate models using the mobile application. These are then uploaded to the system for approval and become pending models. These pending models then become public for other users to view and vote upon. Users then submit votes back to the server. As data is collected, the server continually analyzes votes for pending models. When a specific model passes approval, the server will combine that model into a newly approved model that will be viewable by the public. Users will then be able to continue refining the approved model.

The model in Fig. 2 was contributed by 2 users and took at total of 25 minutes to complete. This model includes 3D structures representing 6 classrooms (with chairs and desks), hallways, restroom areas, doors, and exits.

\section{IMPLEMENTATION DETAILS}

The mobile application was developed on iOS for the iPhone, iPod, and iPad. 3D rendering is utilized through OpenGL ES and GL shader language to efficiently draw and visualize building geometry. In our current implementation we store building geometry in Collada![1], an open format, which uses XML schema for transporting 3D assets between multiple applications. The format also provides a hierarchy that allows for relationships between objects.

The mobile devices communicate over HTTP with an Apache webserver, which connects to a MySQL database. The webserver periodically runs the approval process, pulling new votes from the MySQL database. The server systems are written in PHP to send and receive data to the mobile devices. The geometric data sent from the server is compressed on the fly using gzip compression to maximize bandwidth.

\section{Demo Setup Requirements}

In our demo, we will involve conference attendees in using our crowdsourcing application to model the conference venue and to vote on constructed models. To do so, we will make the crowdsourcing application available in advance for download to conference attendees' mobile phones. As the conference begins, we will encourage attendees to use the application to create and submit 3D geometric models that represent various parts of the conference venue. We will provide a website that shows the progress of the conference 


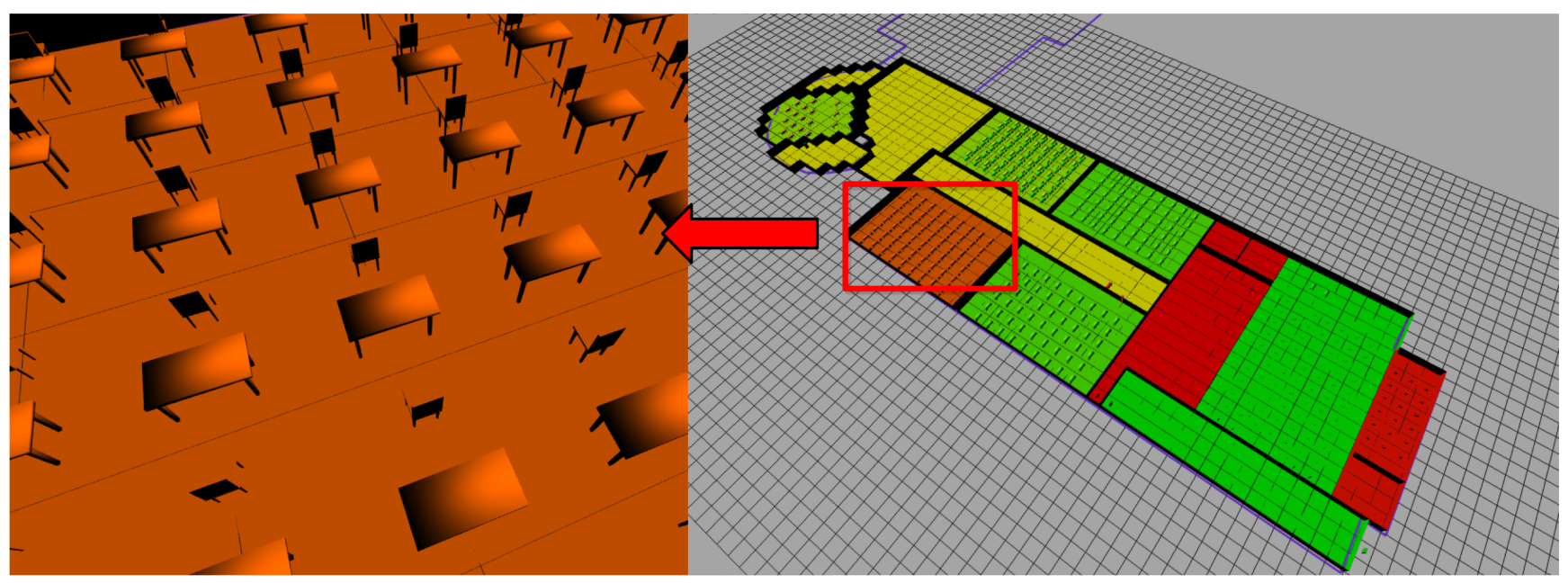

Figure 2. Model of the first floor of an academic building. The building contains 6 classrooms with furniture (see Inset on the left), hallways, restroom areas. Coloring corresponds to approval of the model components(Red has the fewest and green the highest number of votes).

venue being mapped in real time and displays a "leader board" to motivate users to contribute models. During the live demo session, we will show attendees the models that have been submitted, a visualization that overlays the provided models over a manually constructed model of the ground truth of the conference venue, and the votes that have been received. We will also encourage attendees to use mobile phones that are pre-loaded with our application (we will provide and control these at the demo table) to vote on constructed models, and will encourage them to download the application on their own phones.

In terms of technical requirements to support our demo, the crowdsourcing application requires WiFi to connect to the Internet in order to communicate with the server. The server will be hosted by our institution. We will use two laptops at the demo table, one to display the models, and another to display a visualization of the accuracy of the crowdsourced models; a power source is needed for these machines.

\section{CONCLUSION}

This demo paper presents preliminary work on a mobile application for constructing 3D models of buildings by exploiting crowdsourcing to contribute and model quality. We believe this approach has tremendous value, given the success of crowdsourcing in other domains and the fact that the mobile platform continues to improve in terms of computing power, storage and graphics rendering capabilities. We are currently working on improving usability of the mobile application interface to further simplify the creation of 3D geometry. In future work, we plan to improve and optimize the approval process and to incorporate incentives that might be helpful in engaging users to participate in such joint efforts. Finally, we are planning studies that will evaluate the feasibility of this approach and the accuracy of crowdsourced models; in fact, this demo provides an opportunity to collect data that can be used for formative and summative evaluation of our system.

\section{REFERENCES}

[1] Collada, "Digital asset and fx exchange schema," http://collada.org.

[2] M. Goodchild, "Citizens as sensors:the world of volunteered geography," GeoJournal, vol. 69, no. 4, pp. 211-221, 2007.

[3] J. Guest, T. Eaglin, K. Subramanian, and W. Ribarsky, "Visual analysis of situationally aware evacuations," in Proceedings of SPIE Conference on Visualization and Data Analysis, Feb. 2013, to appear.

[4] M. Haklay and P. Weber, "Openstreetmap: User-generated street maps," Pervasive Computing, vol. 7, no. 4, pp. 12-18, Dec. 2008.

[5] J.Liu, K.Lyons, K. Subramanian, and W. Ribarsky, "Semiautomated processing and routing within indoor structures for emergency response applications," in Proceedings of SPIE Conference on Defense, Security, and Sensing, Apr. 2010.

[6] S. Kim, A. Mellama, D. Ebert, and T. Collins, "Visual analytics on mobile devices for emergency response," in Proceedings of IEEE Symposium on Visual Analytics and Technology(VAST), Oct. 2007, pp. 35-42.

[7] A. Rice and O. Woodman, "Crowd-sourcing world models with openroommap," in Eigth annual IEEEE international conference on Pervasive Computing and Communication(Percom) Work-in-Progress, Mar. 2010.

[8] wikipedia, http://en.wikipedia.org.

[9] O. Woodman and R. Harle, "Pedestrian localisation for indoor environments," in Proceedings of the 10th international conference on Ubiquitous computing, 2008, pp. 114-123. 